\title{
O efeito do alerta emocional na qualidade da memória
}

\section{The effect of emotional arousal in the quality of memory}

\author{
Carmem Beatriz NEUFELD \\ Priscila Goergen BRUST-RENCK² \\ Liziane Souza LEITE ${ }^{1}$ \\ Priscila de Camargo PALMA'
}

\begin{abstract}
Resumo
Este artigo visa fornecer evidências científicas da susceptibilidade das memórias à distorção mediante um estudo das falsas memórias (lembranças de eventos que, na realidade, não ocorreram) para eventos complexos estimulantes. A amostra foi composta por 380 adultos com idade entre 17 e 45 anos. Foi usada a versão brasileira do Procedimento de Apresentação de Slides de Cahill e McGaugh adaptada por Neufeld, Brust e Stein para a realidade Sul-brasileira e para a investigação do efeito do alerta emocional na memória e nas falsas memórias. O procedimento é constituído por 11 slides, acompanhados por duas versões de uma narrativa (uma emocionalmente estimulante, sendo então a versão estimulante, e outra com um apelo emocional muito menos intenso, ou seja, a versão controle). O desempenho da memória foi avaliado por meio de um teste de memória de reconhecimento. Os resultados indicaram que a introdução de informações emocionais no meio da versão emocionalmente estimulante pode levar ao aumento de memórias verdadeiras, bem como ao aumento da produção de falsas memórias.
\end{abstract}

Unitermos: Falsas memórias; Histórias; Memória; Nível de alerta.

\begin{abstract}
This study extends scientific evidence on the susceptibility of distorted memories by assessing false memories (remembering events that did not actually occur) in emotionally arousing complex events. The experiment was conducted with 380 participants, aged between 17 and 45 years. The South Brazilian version of Cahill and McGaugh's Slideshow Procedure adapted by Neufeld, Brust and Stein on the evaluation of the effect of emotion on memory and false memories was used. The procedure entailed showing the participant 11 slides, followed by asking the participant to read one of the two possible versions of a narrative (either the emotionally arousing or neutral narrative). Memory performance was investigated using a recognition memory test. Results suggest that introducing emotional information into the arousing narrative may lead to the enhancement of true memory as well as to the enhancement of the production of false memories.
\end{abstract}

Uniterms: False memories; Stories; Memory; Arousal.

Pesquisas indicam que as informações emocionais tendem a influenciar a memória, causando impacto importante e imediato, muitas vezes responsável por distorções que acarretam prejuízos à vida das

\section{$\boldsymbol{\nabla} \mathbf{v} \boldsymbol{\nabla}$}

1 Universidade de São Paulo, Faculdade de Filosofia, Ciências e Letras, Departamento de Psicologia, Programa de Pós-Graduação em Psicologia. Av. Bandeirantes, 3900, 14040-901, Ribeirão Preto, SP, Brasil. Correspondência para/Correspondence to: C.B. NEUFELD. E-mail: <cbneufeld@usp.br>

2 Cornell University, Departament of the Human Development. Ithaca, NY, United States.

Apoio: Fundação Araucária. 
pessoas (Anderson, Yamaguchi, Grabski \& Lacka, 2006; Reisberg \& Heuer, 2004). Um dos principais tópicos de interesse sobre distorções mnemônicas é o fenômeno das falsas memórias. Este fenômeno consiste na recordação de eventos que jamais aconteceram, ou eventos que ocorreram de maneira diferente da recuperada (Brainerd \& Reyna, 2005; Neufeld, Brust \& Stein, 2010; Stein \& Neufeld, 2001).

Uma das teorias que melhor explica este fenômeno é a Teoria do Traço Difuso (Reyna \& Brainerd, 1995), para a qual a memória não é um sistema unitário, existindo dois sistemas independentes de memória que são processados paralelamente: a memória da essência e a memória literal. A memória de essência é ampla, robusta e armazena as informações inespecíficas, ou seja, aquelas que representam o significado da experiência como um todo. Já a memória literal codifica as informações de forma precisa, de modo que os detalhes são registrados e armazenados como episódios, sendo, contudo, mais suscetível ao esquecimento e à interferência, se comparada à memória de essência.

Pesquisadores (Hudson, Fivush \& Kuebli, 1992; Raskin \& Esplin, 1991) acreditam que tarefas excessivamente fáceis não utilizariam recursos atencionais suficientes para serem memoráveis, gerando, assim, um decréscimo na acuracidade da memória para eventos cotidianos e menos importantes. A partir disso, falsas memórias se restringiriam a fatos periféricos da vida das pessoas. Assim, as memórias relacionadas a situações traumáticas ou emocionalmente carregadas estariam imunes a esses erros de memória, devido aos recursos atencionais depositados em tais situações.

O impacto da emoção na memória tem sido estudado em muitos casos por meio de materiais como palavras semanticamente associadas em testes realizados imediatamente após sua apresentação, como no estudo de Corson e Verrier (2007). No entanto, na vida real, as situações envolvem cenários complexos que são avaliados dias ou meses após o evento. Pesquisas com materiais complexos, como o Procedimento de Apresentação de Slides de Cahill e McGaugh (1995), têm sugerido que as informações de eventos estimulantes são mais bem recuperadas (Quevedo et al., 2003). No entanto, esses estudos não avaliaram as distorções 338 mnemônicas.
A emoção está presente em experiências que podem suscitar desde respostas intensas, porém de duração relativamente curta, até reações constantes a estímulos específicos (Pergher, Grassi-Oliveira, Ávila \& Stein, 2005). Um estímulo emocional pode ser caracterizado por diversas dimensões, dentre as quais se observa o alerta e a valência. $\bigcirc$ alerta se refere à ativação gerada pelo estímulo, ou seja, a um conjunto de respostas cognitivas e fisiológicas que preparam o organismo para alguma determinada ação e interação social, sendo um contínuo que pode ir da calma à excitação. Assim, um estímulo alerta pode ser relaxante ou estimulante. A valência, por sua vez, refere-se ao conteúdo do estímulo, caracterizando-se por um contínuo que vai do agradável ao desagradável, e se divide em positiva, neutra e negativa (Barrett \& Russell, 1999).

O impacto da valência e do alerta na memória tem sido alvo de inúmeras discussões na tentativa de identificar a dimensão responsável pela melhor retenção de informações na memória (Kensinger, 2009). Corson e Verrier (2007), por exemplo, relatam os efeitos do alerta no desempenho da memória. Os autores utilizaram dez listas de Palavras Associadas (Roediger \& McDermott, 1995) para investigar os efeitos de alerta e valência em suas combinações possíveis (positivo-estimulante; positivo-relaxante; negativo-estimulante; negativo-relaxante; neutro-neutro). Os resultados demonstram índices de falsas recordações superiores para os grupos que receberam as listas de palavras estimulantes (tanto positiva como negativa) do que para os que receberam as listas relaxantes ou neutras. Não foram encontradas diferenças nas falsas recordações entre os grupos estimulantes nem entre os relaxantes, indicando que o alerta, e não a valência, seria responsável pelo aumento das falsas memórias.

O papel do alerta na memória, no entanto, parece ser alvo de discordância na literatura. Enquanto alguns pesquisadores acreditam que a presença do alerta gera mais lembranças verdadeiras e que as memórias relacionadas estariam imunes a erros de memória (McGaugh, 2000), outros estudos sugerem que as distorções mnemônicas estão presentes inclusive em situações traumáticas ou emocionalmente carregadas (Rohenkohl, Gomes, Silveira, Pinto \& Santos, 2010). 0 caráter traumático de tais eventos revela implicações 
para as áreas clínica (Mazzoni, Loftus \& Kirsch, 2001) e jurídica (Lindsay, Allen, Chan \& Dahl, 2004), sugerindo que nem sempre as lembranças discutidas em terapia ou em juízo são verídicas.

Atualmente diversos estudos são realizados na tentativa de aumentar a efetividade dos instrumentos para mensurar a emoção, na tentativa de melhorar o entendimento a respeito de seus efeitos na memória (Santos, Silveira, Gomes \& Stein, 2009). Estudos com instrumentos complexos como o procedimento de apresentação de slides parecem contribuir para o entendimento dos mecanismos envolvidos na lembrança de informações emocionalmente estimulantes (Maheu, Joober, Beaulieu \& Lupien, 2004; Neufeld, Brust \& Stein, 2008b). No Brasil, a recente adaptação do instrumento por Neufeld et al. (2008a) foi um passo na direção de disseminar pesquisas com maior validade ecológica. No entanto, ainda se faz necessário expandir a investigação para uma amostra maior, equilibrada em termos de diferenças de sexo e faixa etária.

Andreano, Arjomandi e Cahill (2008) revisaram a existência da diferença dos sexos no desempenho da memória emocional, evidenciando que mulheres são mais susceptíveis a esquecimento para eventos emocionalmente carregados. Embora ainda sejam poucos os estudos que ressaltam este fato (Cahill, Gorski, Belcher \& Huynh, 2004; Cahill \& van Stegeren, 2003), os autores enfatizam a influência do período do ciclo menstrual no desempenho da memória para eventos emocionais. Ferree, Kamat e Cahill (2011), inclusive, ressaltam que mulheres recuperam mais intrusões do que homens para eventos emocionalmente estimulantes. No entanto, os mecanismos envolvidos neste fenômeno ainda não se encontram suficientemente explicados. Uma possível hipótese pode ser a faixa etária, uma vez que a oscilação hormonal estabiliza com o passar do tempo.

Este trabalho expandiu a amostra utilizada por Neufeld et al. (2008a) com o intuito de investigar o efeito do alerta emocional de situações complexas na qualidade da memória. Além disso, dando continuidade aos estudos de Neufeld et al. (2008a), considerou as possíveis diferenças relativas a sexo e idade na qualidade da memória, utilizando-se do mesmo instrumento e procedimentos do estudo anterior.

\section{Método}

O estudo envolveu um delineamento fatorial misto $2 \times 2 \times 3 \times 3$ com medidas repetidas nas duas últimas variáveis. A primeira variável, versão da história, foi manipulada intergrupos, sendo que parte dos participantes assistiu à versão estimulante e outra parte, à versão controle. A segunda variável, sexo, ficou distribuída homogeneamente entre os dois grupos. Já no que se refere às duas últimas variáveis, ambas foram manipuladas intragrupos. Todos os participantes receberam material-alvo dividido em três fases e três tipos de itens no teste de memória (alvo, distrator relacionado e distrator não relacionado). As variáveis dependentes foram o desempenho da memória e a atribuição subjetiva do nível de emoção despertada pelo materialalvo.

\section{Participantes}

Participaram do estudo 380 estudantes universitários, de diversos cursos de graduação, com idade entre 17 e 45 anos ( $M=29,1 ; D P=10,06)$. A versão com menos apelo emocional da história (con-trole) foi apresentada para 193 participantes $(M=29,8 ; D P=9,83)$, sendo 103 do sexo feminino e 90 do masculino, enquanto 187 assistiram à versão estimulante, com intenso apelo emocional ( $M=28,37 ; D P=10,28)$, sendo 106 do sexo feminino e 81 do masculino. Todos os participantes foram selecionados por conveniência e foram designados aleatoriamente para assistir a uma ou outra versão da história.

\section{Instrumentos}

O material a ser memorizado foi o Procedimento de Apresentação de Slides de Cahill e McGaugh (1995), cujas imagens foram adaptadas para a realidade Sul-brasileira por Neufeld et al. (2008a) e as narrativas foram traduzidas para a língua portuguesa por Quevedo et al. (2003). O material foi constituído por uma sequência de 11 imagens acompanhadas por duas versões de narrativas: uma controle, com um apelo emocional menos intenso, e a outra experimental, ou seja, estimulante, com um apelo emocional mais intenso. As duas versões da história foram gravadas em CD-Rom, narradas por uma estudante de comunicação social, com o cuidado 
de manter fixo o intervalo de 6 segundos para a apresen-tação de cada slide.

As versões da história foram divididas em três fases, de forma que a fase 1 (slides 1 a 4) apresentava uma mãe e seu filho a caminho do hospital e a foto do pai em um laboratório, a fase 2 (slides 5 a 8) apresentava um carro batido e diversos procedimentos hospitalares, enquanto a fase 3 (slides 9 a 11) apresentava a mãe caminhando na rua, ao telefone e indo embora. A mesma sequência de slides é apresentada para cada versão da história que se diferem em função da narrativa. As narrativas referentes às fases 1 e 3 também eram idênticas para os dois grupos, e a fase 2 foi responsável pela diferenciação da emocionalidade entre as versões da história. A fase 2, também denominada fase crítica, relatava ou que houve um acidente que causara graves ferimentos no menino (versão estimulante), ou que o menino viu um carro batido e assistiu a um treinamento de atendimento de urgência no hospital onde o pai trabalhava.

A emoção despertada pela história foi medida por meio de uma escala Likert que consistia de duas questões, semelhante ao estudo de Neufeld et al. (2008a). Os participantes deveriam, inicialmente, indicar se a história foi responsável por gerar alguma emoção neles por meio de uma escala dicotômica "sim"ou "não". Em caso afirmativo, eles deveriam indicar o nível de intensidade da emoção sentida frente à versão da história em uma escala Likert de cinco pontos, variando de "quase nenhuma" (1) até "extrema" (5).

O teste de memória de reconhecimento desenvolvido por Neufeld et al. (2008a) foi aplicado. O teste era composto por 25 itens de escolha simples, sobre os quais os participantes deveriam informar se correspondiam às informações que eles haviam visto e ouvido na história. Os itens foram distribuídos aleatoriamente ao longo do teste em quantidades proporcionais para as três fases da história. As diferenças na fase 2 foram consideradas de forma que as questões referentes a cada versão encontravam sua alternativa correspondente no teste para a outra versão. Assim, para o item "o menino sofre um grave acidente" do teste para a versão estimulante havia a alternativa "o menino viu um carro batido" no teste para a versão controle.

As questões do teste pertenciam a três categorias de resposta: alvo, distrator relacionado e distrator não relacionado. Os itens-alvo referiam-se a informações que estavam presentes na história, portanto, verdadeiras, como "a mãe tranca a porta antes de sair de casa"; os distratores relacionados referiam-se a informações que estavam relacionadas a informações da história, mas que não eram corretas, como "o carro era vermelho" (quando, na realidade, era preto); e os distratores não relacionados referiam-se a informações que não estavam relacionadas à história, como "a tia do menino trabalha em um escritório".

\section{Procedimentos}

O estudo foi submetido à apreciação do Comitê de Ética em Pesquisa com seres humanos, sendo sua coleta de dados iniciada apenas após a aprovação do Protocolo Ofício 720/04 pelo Comitê de Ética em pesquisa/Pontifícia Universidade Católica do Rio Grande do Sul. Os procedimentos relativos à ética na pesquisa foram tomados em conformidade com a legislação nacional.

A coleta de dados foi realizada em sala silenciosa, com a utilização de equipamento de multimídia para projeção dos slides e aparelho de som para reprodução de uma ou outra versão da história. Após uma explicação do estudo e de assegurada a total liberdade para o participante desistir a qualquer momento da pesquisa, um Termo de Consentimento Livre e Esclarecido foi assinado por todos os que consentiram em participar.

Foi solicitado aos participantes que prestassem atenção na sequência de slides e na narrativa, frisando a importância de os mesmos evitarem fazer comentários ao longo dos procedimentos, com o intuito de não desviarem a atenção. Logo após a apresentação de uma ou outra versão da história, os participantes responderam à escala subjetiva de emoção, na qual lhes foi solicitado responder com o máximo de sinceridade sobre a intensidade que atribuíam à emoção despertada pela história assistida.

Ao final, os participantes respondiam ao teste de memória de reconhecimento autoadministrado. A instrução solicitava que as respostas fossem baseadas apenas na memória para a história assistida, de forma que sempre que o participante lembrasse uma informação que havia sido apresentada, deveria assinalar a opção "sim"; caso contrário, deveria assinalar a opção 
"não". Foi apresentado um item exemplo instruindo o participante sobre como responder ao teste. Foi também solicitado ao participante que respondesse às questões na ordem em que elas aparecessem no teste, não deixando nenhuma questão em branco, nem retornando às questões anteriores.

\section{Resultados e Discussão}

\section{Indicadores de emocionalidade}

Com o objetivo de verificar o efeito da emocionalidade da narrativa na memória, foi conduzido um teste $t$ de Student para testar o efeito entre a escala dicotômica de emoção e as versões da história. Por meio dessa análise $(t(378)=7,57 ; p<0,001)$ observou-se que os participantes que assistiram à versão estimulante $(M=0,82 ; D P=0,38)$ identificaram um impacto emocional desencadeado pela história maior do que os que assistiram à versão controle $(\mathrm{M}=0,48 ; \mathrm{DP}=0,5)$.

Esse resultado foi qualificado pelo nível de intensidade da emoção de todos os participantes que se sentiram impactados emocionalmente pela história, por meio de um teste tentre a escala subjetiva de emoção e as versões da história. Nesta análise foi evidenciado um efeito principal de grupo ( $t(244)=7,2$; $p<0,001)$, tendo a história sido classificada como mais emocional pelos participantes que assistiram à versão estimulante $(M=3,14 ; D P=0,84)$ do que pelos que assistiram à versão controle $(M=2,3 ; D P=0,94)$. Esses dados sugerem que o material-alvo estimulante despertou maior intensidade de emoção do que o controle, corroborando os demais estudos na área (Canli, Zhao, Brewer, Gabrieli \& Cahill, 2000).

\section{Indicadores de memória}

Os resultados foram analisados com base nas três possibilidades de resposta do teste de memória: alvo, distrator relacionado ou distrator não relacionado. Um item alvo referia-se a uma medida de memórias verdadeiras, um distrator relacionado referia-se a uma medida de falsas memórias, enquanto um distrator não relacionado era considerado uma resposta de viés ou "chute".
Os dados foram analisados com o auxílio do programa Statistical Package for Social Sciences (SPSS) versão 15.0. Todos os tratamentos estatísticos utilizaram um $\alpha=0,05$ para o teste de hipóteses. Foram realizadas análises post hoc e comparações pareadas com correção de Bonferroni. Como não foram observadas diferenças entre os participantes homens e mulheres, esta variável foi excluída de todas as análises.

A avaliação do desempenho da memória foi realizada por meio de uma Análise de Variância (Anova) entre as duas versões da história com medidas repetidas para memória verdadeira e falsas memórias ao longo das três fases da história, de forma que foi observada uma interação de todas as variáveis $(F(2,239)=9,97$; $p<0,001)$. Esse resultado foi qualificado por uma análise post hoc destacada na Tabela 1, que sugere que os índices de reconhecimento das Memória Verdadeira (MV) foram superiores para os participantes que assistiram à versão controle do que para os que assistiram à versão estimulante na primeira fase da história $(p<0,05)$. Este achado vai de encontro com o estudo de Gasbarri et al. (2005), que sugere que o alerta emocional potencializa a recuperação das informações, especialmente para a segunda fase da história. Por outro lado, a presença de um fator emocional na segunda fase da versão estimulante pode direcionar o foco da atenção do participante de forma a gerar uma interferência retroativa sobre as informações da fase 1.

Outro olhar sobre a Anova traz luz a essa questão ao demonstrar que mais falsas memórias foram produzidas na segunda fase da história pelos participantes que assistiram à versão estimulante em relação aos que assistiram à versão controle $(p<0,001)$, conforme os dados da Tabela 1. Esse resultado corrobora o estudo de revisão de Van Giezen, Arensman, Spinhoven e Wolters (2005), que enfatiza que a emocionalidade do evento interfere na consolidação da memória, podendo gerar distorções. Como as respostas de viés - que não estavam relacionadas a nenhuma fase da história, serviam apenas como indicativo do tipo de resposta que o participante produzia no teste, no intuito de verificar se estava prestando atenção no momento da realização da tarefa - não apresentaram alterações entre as versões da história, foram excluídas das próximas análises. 
Tabela 1

Média de reconhecimento por tipo de item, fase e versão da história

\begin{tabular}{|c|c|c|c|c|}
\hline \multirow{2}{*}{ Fase da história } & \multicolumn{2}{|c|}{ Memória verdadeira } & \multicolumn{2}{|c|}{ Falsas memória } \\
\hline & Controle & Experimental & Controle & Experimental \\
\hline 1 & $0,66 \quad(0,29)^{*}$ & $0,59(0,30)$ & $0,50(0,40)$ & $0,47(0,38)$ \\
\hline 2 & $0,84 \quad(0,24)$ & $0,88(0,20)$ & $0,29(0,36)$ & $0,49(0,35)^{* * *}$ \\
\hline 3 & $0,53 \quad(0,29)$ & $0,50(0,30)$ & $0,52(0,35)$ & $0,49(0,34)$ \\
\hline Total & $0,69 \quad(0,20)$ & $0,68(0,18)$ & $0,44(0,23)$ & $0,48(0,23)$ \\
\hline
\end{tabular}

Nota: ${ }^{*} p<0,05$ entre versões da história; ${ }^{* *} p<0,001$ entre versões da história. Desvio-padrão entre parênteses.

Uma análise dos resultados entre as fases da história foi realizada segundo o modelo de Cahill e van Stegeren (2003) usando o mesmo procedimento. Os autores calcularam o índice da diferença da recuperação das informações da fase 2 em relação às da fase 1 de ambas as versões da história. Dessa forma, é possível identificar o alerta emocional na memória para a fase em que as informações emocionais são introduzidas (fase 2) em comparação à fase neutra inicial quando os elementos da história são introduzidos (fase 1).

A diferença do desempenho da memória para as informações da fase 2 em relação às da fase 1 foi avaliada por meio de uma Anova entre as versões da história, em que foi possível identificar uma interação $(F(1,378)=4,04 ; p<0,05)$. Esse resultado sugere que os participantes que assistiram à versão estimulante lembraram mais informações na fase 2 da história em relação à fase $1(M=0,29 ; D P=0,32)$ do que os participantes que assistiram à versão controle $(M=0,18$; $\mathrm{DP}=0,32 ; p<0,01)$. Esse resultado corrobora a hipótese de McGaugh (2000) de que a emoção, representada pelas informações estimulantes introduzidas na fase 2 , potencializa a memória verdadeira com o passar do tempo.

Por outro lado, mais falsas memórias foram produzidas na segunda fase da história em relação à primeira pelos participantes que assistiram à versão estimulante ( $M=0,02 ; \mathrm{DP}=0,47$ ) do que pelos que assistiram à versão controle $(M=-0,21 ; D P=0,48 ; p<0,001)$. 0 efeito do alerta emocional na memória, portanto, parece ser controverso como sugerem Porter, Spencer e Birt (2003), uma vez que, ao mesmo tempo em que gera o aumento de lembranças verdadeiras quando informações estimulantes são introduzidas na história, produz mais falsas memórias. Tais resultados são consonantes com os encontrados por Neufeld et al. (2008a), indicando que os mecanismos referentes aos efeitos do alerta emocional sobre a memória e sua distorção parecem aumentar na recuperação de informações, mesmo que estas não sejam, necessariamente, embasadas em informações vivenciadas pelo indivíduo.

Ao contrário do que ressaltado na literatura sobre memória emocional (Andreano, Arjomandi \& Cahill 2008; Ferree et al., 2011), não foram observadas diferenças no desempenho da memória de homens e mulheres $(p>0,05)$, inclusive quando se levou em consideração a faixa etária dos participantes $(p>0,05)$. Por outro lado, esse resultado vai ao encontro dos estudos de falsas memórias que enfatizam um desempenho semelhante na população adulta, variando apenas em crianças e idosos (Brainerd, Reyna \& Ceci, 2005). O novo material parece, portanto, ser mais sensível à avaliação do desempenho da memória e das falsas memórias em adultos, independentemente do sexo e idade dos participantes.

\section{Considerações Finais}

O objetivo deste trabalho foi investigar o impacto da emoção na memória para eventos complexos com apelo emocional. Para isso, principiou-se pela comparação do desempenho da memória de homens e mulheres adultos em diferentes faixas etárias entre duas versões de uma mesma história (uma estimulante e outra neutra). Em geral, os resultados indicaram que não foram encontradas diferenças nos índices de recordação verdadeira ou falsa, ainda que alguns estudos apresentem diferenças na recuperação de informações emocionais entre homens e mulheres. Esse resultado parece ser em função de o teste de memória ter sido 
aplicado imediatamente após a apresentação do material-alvo. A consolidação da memória verdadeira para situações alerta é moderada pelos efeitos da amígdala no ser humano, mas somente para informações recuperadas horas ou dias após a exposição ao material-alvo. O momento da testagem, portanto, parece influenciar o desempenho da memória para informações estimulantes verdadeiras.

Por outro lado, no experimento realizado foi possível observar o efeito do alerta emocional no desempenho da memória verdadeira, uma vez que mais informações foram lembradas na fase 2 em comparação à fase 1 da versão estimulante do que da versão controle quando o escore diferencial foi calculado. Esse resultado é consistente com os estudos de memória emocional que enfatizam o fato de o caráter estimulante de um evento ser responsável pelo aumento do desempenho da memória.

A investigação da produção de falsas memórias também demonstrou um aumento na fase estimulante (fase 2) da versão estimulante da história quando o escore diferencial foi calculado em comparação à versão controle. Esse resultado corrobora dados de pesquisas experimentais sobre falsas memórias e alerta emocional. O aumento das falsas memórias também foi observado na mesma direção quando comparado o escore bruto entre as fases emocionais das duas histórias. Nesse caso, no entanto, observou-se a ausência do impacto da emoção na memória verdadeira.

Portanto, em geral, o estudo reforça o que foi observado por outros estudos, indicando que eventos emocionais são relembrados em maior quantidade, porém podem ser igualmente falsificados. Em outras palavras, ao mesmo tempo em que o alerta emocional interfere na consolidação da memória, podendo gerar falsas memórias, também serve como uma proteção da memória, mantendo memórias verdadeiras.

Conclui-se, portanto, que a versão brasileira do Procedimento de Apresentação de Slides logra êxito na investigação da influência do alerta emocional na memória, bem como das falsas memórias. Uma vantagem da utilização de um instrumento que simula uma cena real é o grau de verossimilhança dos resultados, o que permite uma consideração a respeito da validade do estudo sobre a precisão da memória.
Apesar disso, o procedimento apresenta algumas limitações, como a perda de controle experimental em virtude da repetida exposição de informações das imagens. As informações repetidas tendem a ser mais resistentes ao esquecimento, bem como à falsificação na memória. Neste caso, a mãe e a criança, que aparecem respectivamente em seis e três slides, seriam lembradas com maior precisão. A repetição também impede a identificação da fase da história à qual pertence a informação recuperada. Assim, embora o Procedimento de Apresentação de Slides permita uma avaliação do impacto do alerta na memória para eventos complexos, simulando uma situação real, uma avaliação mais precisa deste impacto pode ser investigada por meio de estudos com palavras semanticamente associadas.

Por fim, os resultados obtidos reforçam a discussão sobre as implicações em áreas aplicadas da Psicologia e da saúde em geral. Os temas tratados pelos psicólogos (clínicos ou forenses) em geral carregam forte apelo emocional, girando em torno de uma semântica comum. Tais características podem ser consideradas similares às condições manipuladas no estudo experimental descrito neste artigo. No entanto, cabe lembrar que a amostra deste trabalho foi composta por estudantes universitários, e não foi realizada nenhuma testagem psicológica no intuito de verificar a presença ou não de sintomas ou transtornos mentais. Sugere-se que estudos futuros testem o procedimento em população clínica, no intuito de verificar se a presença de sintomas e/ou transtornos mentais exerce efeito sobre a memória de eventos estimulantes.

\section{Referências}

Anderson, A. K., Yamaguchi, Y., Grabski, W., \& Lacka, D. (2006). Emotional memories are not all created equal: Evidence for selective memory enhancement. Learning \& Memory, 13(6), 711-718.

Andreano, J., Arjomandi, H., \& Cahill, L. (2008). Menstrual cycle modulation of the relationship between cortisol and long-term memory. Psychoneuroendocrinology, 33(6), 874-82.

Barrett, L. F., \& Russell, J. A. (1999). The structure of current affect: Controversies and emerging consensus. Current Directions in Psychological Science, 8(1), 10-14.

Brainerd, C. J., \& Reyna, V. F. (2005). The science of false memory. New York: Oxford University. 
Brainerd, C. J., Reyna, V. F., \& Ceci, S. J. (2005). Developmental reversals in false memory: A review of data and theory. Psychological Bulletin, 134(3), 343-382.

Brainerd, C. J., Stein, L. M., Silveira, R. A., Rohenkohl, G., \& Reyna, V. F. (2008). How does negative emotion cause false memories? Psychological Science, 19(9), 919-925.

Cahill, L., Gorski, L., Belcher, A., \& Huynh, Q. (2004). The influence of sex versus sex related-traits on long-term memory for gist and detail from an emotional story. Consciousness and Cognition, 13(2), 391-400.

Cahill, L., \& McGaugh, J. L. (1995). A novel demonstration of enhanced memory associated with emotional arousal. Consciousness and Cognition, 4(4), 410-421.

Cahill, L., \& van Stegeren, A. (2003). Sex-related impairment of memory for emotional events with beta-adrenergic blockade. Neurobiology of Learning and Memory, 79(1), 81-88.

Canli, T., Zhao, Z., Brewer, J., Gabrieli, J. D. E., \& Cahill, L. (2000). Event-related activation in the human amygdala associates with later memory for individual emotional experience. The Journal of Neuroscience, 20(19), 1-5.

Corson, Y., \& Verrier, N. (2007). Emotions and false memories: Valence or arousal? Psychological Science, 18(3), 208-211.

Ferree, N., Kamat, R., \& Cahill, L. (2011). Influences of menstrual cycle position and sex hormone levels on spontaneous intrusive recollections following emotional stimuli. Consciousness and Cognition, 20(4), 1154-1162.

Gasbarri, A., Pompili, A., Arnone, B., d'Onofrio, A., Marchetti, A., Tavares, M. C., et al. (2005). Declarative memory retention and emotional stimuli: A study of an Italian sample. Functional Neurology, 20(4), 157-162.

Hudson, J., Fivush, R., \& Kuebli, J. (1992). Scripts and episodes: The development of event memory. Applied Cognitive Psychology, 6(6), 483-505.

Kensinger, E. A. (2009). Remembering the details: Effects of emotion. Emotion Review, 1(2), 99-113.

Lindsay, D. S., Allen, B. P., Chan, J. C. K., \& Dahl, L. C. (2004). Eyewitness suggestibility and source similarity: Intrusions of details from one event into memory reports of another event. Journal of Memory \& Language, 50(1), 96-111.

Maheu, F. S., Joober, R., Beaulieu, S., \& Lupien, S. J. (2004). Differential effects of adrenergic and corticosteroid hormonal systems on human short- and long-term declarative memory for emotionally arousing material. Behavioral Neuroscience, 118(2), 420-428.

Mazzoni, G., Loftus, E. F., \& Kirsch, I. (2001). Changing beliefs about implausible autobiographica events: A little plausibility goes a long way. Journal of Experimental Psychology Applied, 7(1), 51-59.

McGaugh, J. L. (2000). Memory: A century of consolidation. Science, 287(5451), 248-251.

Neufeld, C. B., Brust, P. B., \& Stein, L. M. (2008a). Adaptação de um método de investigação do impacto da emoção na memória. Psico-USF, 13(1), 21-29.

Neufeld, C. B., Brust, P. B., \& Stein, L. M. (2008b). O efeito da sugestão de falsa informação para eventos emocionais: quão susceptíveis são nossas memórias? Psicologia em Estudo, 13(3), 539-547.

Neufeld, C. B., Brust, P. B., \& Stein, L. M. (2010). Compreendendo o fenômeno das falsas memórias. In L. M. Stein (Org.), Falsas memórias: fundamentos científicos e suas aplicações clínicas ejurídicas (pp.21-42). Porto Alegre: Artmed.

Pergher, G. K., Grassi-Oliveira, R., Ávila, L. M., \& Stein, L. M. (2005). Memória, humor e emoção. Revista de Psiquiatria do Rio Grande do Sul, 28(1), 5-12.

Porter, S., Spencer, L., \& Birt, A. R. (2003). Blinded by emotion? Effect of the emotionality of a scene on susceptibility to false memories. Canadian Journal of Behavioural Science, 35(2), 165-175.

Quevedo, J., Sant'Anna, M. K., Madruga, M., Lovato, I., deParis, F., Kapczinski, F., et al. (2003). Differential effects of emotional arousal in short and long-term memory in healthy adults. Neurobiology of Learning and Memory, 79(2), 132-135.

Raskin, D., \& Esplin, P. (1991). Assessment of children's statements of sexual abuse. In J. L. Doris (Ed.), The suggestibility of children's recollections (pp.153-164). Washington: American Psychology Association.

Reisberg, D., \& Heuer, F. (2004). Memory for emotional events. In D. Reisberg \& P. Hertel (Eds.), Memory and emotion (pp.3-40). Oxford: Oxford University.

Reyna, V. F., \& Brainerd, C. J. (1995). Fuzzy-trace theory: An interim synthesis. Learning and Individual Differences, $7(1)$, $1-75$.

Roediger, H. L., \& McDermott, K. B. (1995). Creating false memories: Remembering words not presented on lists. Journal of Experimental Psychology: Learning, Memory, and Cognition, 21(4), 803-814.

Rohenkohl, G., Gomes, C. F. A., Silveira, R. A. T., Pinto, L. H., \& Santos, R. F. (2010). Emoção e falsas memórias. In L. M. Stein. Falsas memórias: fundamentos científicos e suas aplicações clínicas e jurídicas (pp.87-100). Porto Alegre: Artmed.

Santos, R. F., Silveira, R. A. T., Gomes, C. F. A., \& Stein, L. M. (2009). Normas de emocionalidade para versão brasileira do paradigma Deese-Roediger-McDermott (DRM). Psicologia: Teoria e Pesquisa, 25(3), 387-394.

Stein, L. M., \& Neufeld, C. B. (2001). Falsas memórias: porque lembramos de coisas que não aconteceram? Arquivos de Ciência Saúde Unipar, 5(2), 179-186.

Van Giezen, A., Arensman, E., Spinhoven, P., \& Wolters, G. (2005). Consistency of memory for emotionally arousing events: A review of prospective and experimental studies. Clinical Psychology Review, 25(7), 935-953.

Welter, C. L. W. (2010). Recordação de eventos emocionais repetitivos: memória, sugestionabilidade e falsas memórias. In L. M. Stein. (Org.), Falsas memórias:fundamentos científicos, aplicações clínicas ejurídicas (pp.186-208). Porto Alegre: Artmed.

Recebido em: 8/9/2011

Versão final em: 4/5/2012

Aprovado em: 21/5/2012 